\title{
On a numerical solution for fuzzy fractional differential equation using an operational matrix method
}

\begin{abstract}
In the current manuscript we suggest an approach to obtain the solutions of the fuzzy fractional differential equations (FFDEs). We found the operational matrix within the modified Laguerre functions. In this way the investigated equations are turned into a set of algebraic equations. We provide examples to illustrate both accuracy and simplicity of the suggested approach.
\end{abstract}

Keyword: Fuzzy fractional differential equations; Laguerre functions; Operational matrices; Numerical methods 